\title{
A Contact Angle Study of the Interaction between Embedded Amphiphilic Molecules and the PDMS Matrix in an Aqueous Environment
}

\section{Wenjun Qiu ${ }^{1}$, Xiaojiao Sun ${ }^{1}$, Chaoqun $\mathrm{Wu}^{2}$, Klas Hjort ${ }^{1}$ and Zhigang Wu ${ }^{1,2, *}$}

1 Department of Engineering Sciences, The Angstrom Laboratory, Uppsala University, S-75105 Uppsala, Sweden; E-Mails: qiuwingqiu85@gmail.com (W.Q.); sun20040807@hotmail.com (X.S.); klas@angstrom.uu.se (K.H.)

2 School of Mechanical \& Electronic Engineering, Wuhan University of Technology, Wuhan 430070, China; E-Mail: chaoqunwu@whut.edu.cn

* Author to whom correspondence should be addressed; E-Mail: zhigang.wu@angstrom.uu.se; Tel.: +46-184-711-086; Fax: +46-184-713-072.

Received: 16 April 2014; in revised form: 3 August 2014 / Accepted: 4 August 2014 /

Published: 7 August 2014

\begin{abstract}
Poly(dimethylsiloxane) (PDMS) surface modification via gradient-induced transport of embedded amphiphilic molecules is a novel, easy, flexible, and environmentally friendly approach for reducing protein adsorption on PDMS in microfluidic applications. To better understand the processing and the potential use in the viability-sensitive applications such as manipulation and culturing of primary neural cells, we systematically investigate how embedded molecules interact with a PDMS matrix and its surface in aqueous environments by studying the wetting angle over time under various processing conditions, including water exposure time, water exposure temperature, curing master materials, in addition to comparing different embedded amphiphilic molecules. The results indicate that the water exposure time clearly plays an important role in the surface properties. Our interpretation is that molecular rearrangement of the surface-embedded molecules improves surface coverage in the short term; while over a longer period, the transport of molecules embedded in the bulk enhance its coverage. However, this improvement finally terminates when molecules transported from the bulk to the surface are not sufficient to replace the molecules leaching into the water.
\end{abstract}


Keywords: gradient-induced transport; PDMS surface modification; embedded amphiphilic molecules

\section{Introduction}

Since Whitesides introduced soft lithography to the community in the 1990s [1], Poly(dimethylsiloxane) (PDMS) has become the most popular material in microfluidics research and prototyping [2-4]. Compared to other materials-silicon, glass or a thermoplastic such as Poly(methylmethacrylate) PMMA-PDMS has many advantages: low cost of both material and fabrication, ease of use, flexibility and good performance in many biological or clinical applications [5]. However, its unstable and hydrophobic surface, in addition to the strong adsorption of biological molecules, hinder its utility in many applications, especially if long shelf times are required. It is therefore important that a good solution for overcoming this drawback is found.

During the past two decades, various approaches have been proposed in reducing biomolecular adsorption on PDMS. These methods can be roughly categorized into three groups: high energy exposure (e.g., plasma and UV exposure), dynamic coating with amphiphilic molecules (surfactants), and chemical grafting of the hydrophilic group. During high energy exposure, the PDMS surface is oxidized and forms a hydrophilic surface with reactive silanol functional groups, which may also be used for further functionalization using organic chemistry $[2,3]$. However, this oxidized surface is not stable in air and cannot survive for long due to hydrophobic surface recovery [6]. In the dynamic coating with amphiphilic molecules, the molecules are in a buffer solution to incubate the surface of PDMS. Hydrophobic interaction is the driving force for the surfactant to be dynamically adsorbed to the surface with their hydrophilic head groups aligned towards the buffer solution. This is a fast and efficient method without strong covalent bonding between surfactants and the PDMS surface [4], but it still has problems since most surfactants reduce the viability and physiological activity of primary cells $[7,8]$. In chemical grafting, a complex process, a polymer with a hydrophilic long chain is covalently tethered onto the surface, which is based on the chemical reaction [2].

As pointed out by Whitesides [5], PDMS is much like a solid solvent, where some organic molecules and solvents have very good solubility [9]. One widely used amphiphilic molecule family is the polaxamers, better known as pluronics, which are common in many areas such as cosmetics, medical applications, tissue engineering, and food additives [10,11]. The molecule has a triblock structure consisting of one hydrophobic polypropylene oxide (PPO) group flanked by two hydrophilic polyethylene oxide (PEO) units. The structure is $\mathrm{PEO}_{n}-\mathrm{PPO}_{m}-\mathrm{PEO}_{n}$, where $\mathrm{n}$ and $\mathrm{m}$ vary in the more than 50 different pluronics available. The hydrophobic PPO chain is attached to a hydrophobic polymer, and the hydrophilic PEO chains self-assemble into a cilia-like surface on the hydrophobic material that effectively repels proteins [10]. The degree of protein repellence depends on the length of the PEO chains and the surface coverage [12-14]. More importantly, pluronics have demonstrated very low toxicity and immunogenic response, and are approved by the U.S. Food and Drug Administration (FDA) in many biomedical applications in vitro and in vivo [15], e.g., drug and gene delivery [16,17]. Recently, more interest was put into applications with cell cultures, due to its low cell toxicity that 
does not hinder the cell viability during the culturing [18]. It can even be used in sensitive mammalian cell cultures, including liver carcinoma cells [19]. Noting the fact that PDMS is a solid solvent [5] and that pluronic has high performance in inhibiting bio-fouling in microfluidic devices [20], we recently presented an inversely dynamic coating with reduced protein adsorption on PDMS [21]. The general process is illustrated in Figure 1. By embedding pluronic molecules inside PDMS, they will migrate towards the surface in an aqueous environment. Hence, part of the pluronic molecules will appear on the surface and reduce biomolecule adsorption as well as enhance wetting. Ultimately, this processing may be used in many high viability sensitive applications such as in studies and manipulation of primary neural cells.

Figure 1. Illustration of the processing of surface modification.
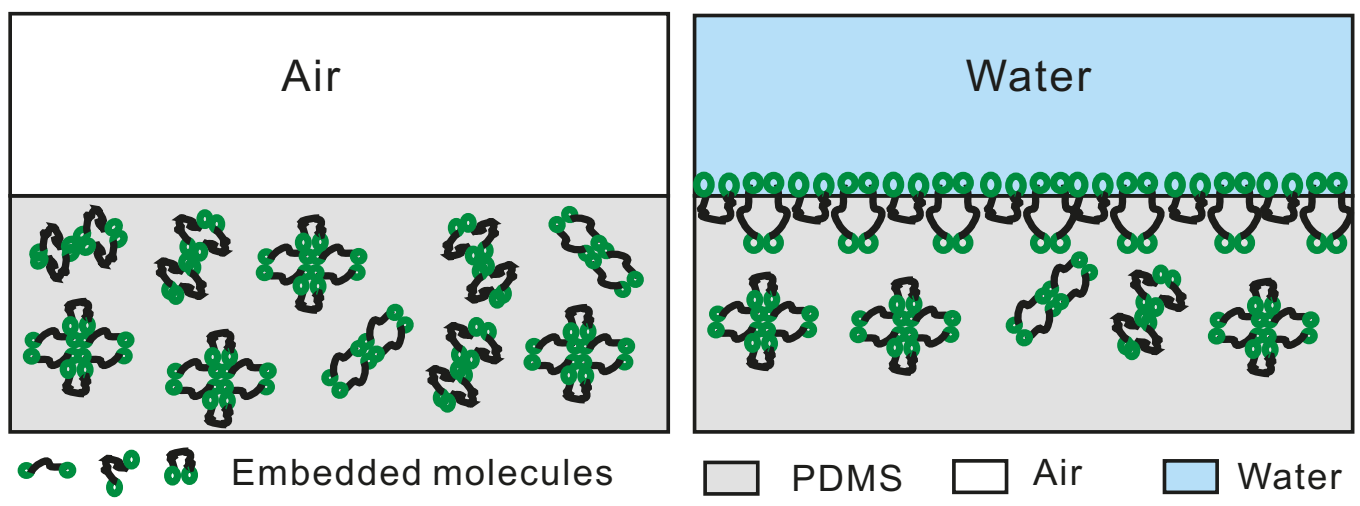

However, how amphiphilic molecules such as pluronic interact with the PDMS matrix and its effects to the surface properties in the short and long term remain unclear. To better understand how the embedded amphiphilic molecules interact with the PDMS matrix in an aqueous environment and to study how they reside on the PDMS surface, how the embedded amphiphilic molecules impact PDMS surface wetting is studied here in detail. In particular, short- and long-term observations of the contact angles were conducted after water exposure. In addition, the dependence of curing master material, water temperature, and the type and size of embedded amphiphilic molecules were investigated.

\section{Experimental Section}

\subsection{Materials}

PDMS (RT601A and B, Wacker Chemie, München, Germany), was prepared by thoroughly mixing the base and curing agent in a ratio of 9:1 (wt:wt). Small amounts of Pluronic F127 and F68 (Sigma-Aldrich, Stockholm, Sweden) were dissolved in ethanol (99.5\%, absolute fine spirit, Kemetyl (Jordbro, Sweden) for saturated solutions $\left(\sim 200 \mathrm{mg} \cdot \mathrm{mL}^{-1}\right)$ separately. Tween 80 (Sigma-Aldrich, Stockholm, Sweden) and PDMS-EO AB111108 (ABCR, Karlsruhe, Germany) were used without pre-treatment. Pluronic F127, F68, Tween 80 and PDMS-EO are non-ionic surfactants. For each surfactant, only a small amount of surfactant was added to the liquid-like uncured PDMS mixture (usually $12.00 \mathrm{~g}$ of PDMS, apart from the one with different master materials): $80 \mu \mathrm{L}$ for Pluronic F127 and F68 solutions while 0.5 wt \% for Tween 80 and PDMS-EO. The mixture of freshly mixed (components A and B) PDMS and the additive surfactant was mixed to obtain uniform distribution of 
the surfactant. Later, the mixture was put in refrigerator for $30 \mathrm{~min}$ to remove bubbles and then in a $75{ }^{\circ} \mathrm{C}$ oven for curing.

\subsection{Master Preparation and PDMS Replica}

In order to investigate how surface configurations change in the curing process, four masters were made of different materials, including a 4" silicon wafer after isopropyl alcohol (IPA) cleaning, a glass (normal glass petri dish, $\sim 4^{\prime \prime}$ ), a polystyrene (normal polystyrene petri dish, $\sim 3.5^{\prime \prime}$, VWR International, Stockholm, Sweden) and PDMS (freshly cross-linked PDMS cured in a polystyrene petri dish). Subsequently, $48.00 \mathrm{~g}$ of uncured PDMS mixture and $320 \mu \mathrm{L} \mathrm{F127}$ solution $\left(\sim 200 \mathrm{mg} \cdot \mathrm{mL}^{-1}\right)$ were mixed thoroughly, and then poured into the aforementioned four masters with the same ratio of weight to bottom surface area, giving the same thickness.

\subsection{Nuclear Magnetic Resonance Spectroscopy}

To study the extracted substance in water, nuclear magnetic resonance spectroscopy (NMR) was used. Firstly, we prepared the sample with $80 \mu \mathrm{L}$ saturated ethanol solution of Pluronic F127 in $10.00 \mathrm{~g}$ thoroughly mixed PDMS prepolymer and $7.50 \mathrm{~g}$ of the above mixture was weighed and cured on a 4 " wafer, as mentioned previously. Four of the samples were then immersed in $500 \mathrm{~mL}$ de-ionized water for $24 \mathrm{~h}$ at room temperature. The immersed water was condensed using a rotary evaporator (Heidolph LABOROTA Efficient T4000, Heidolph Instruments, Schwabach, Germany) and further lyophilized in a freeze dryer (Thermo Scientific, Hetosic, Waltham, MA, USA). Each lyophilized residue was dissolved in $\mathrm{D}_{2} \mathrm{O}$ (Sigma-Aldrich, Stockholm, Sweden) and ${ }^{1} \mathrm{H}$ NMR spectra were recorded at $25{ }^{\circ} \mathrm{C}$ on a $500 \mathrm{MHz}$ spectrometer (Varian, Palo Alto, CA, USA). At the same time, the pure Pluronic F127 was dissolved in $\mathrm{D}_{2} \mathrm{O}$ and ${ }^{1} \mathrm{H}$ NMR spectra were recorded as a reference. As a negative control, four native PDMS samples were prepared correspondingly and immersed in the water. The immersed water was lyophilized and the residue was measured by NMR while processing the samples with Pluronic F127.

\subsection{Contact Angle Measurement}

Wetting characteristics can be measured in many ways and in defining the static contact angle; the static advancing contact angle is most commonly used due to its stability and credibility [22]. However, in this study, due to instrument limitation, the static sessile droplet method was used. Contact angle measurements were performed with a contact angle goniometer (FTA 200, First Ten Angstrom, Portsmouth, VA, USA), which contains an optical system to capture the clear profile of the droplet on a solid substrate, and a computer with a program to analyze the angle. A $2.5 \mu \mathrm{L}$ droplet of de-ionized water was deposited on the solid for each contact angle measurement. The image was captured immediately (within $5 \mathrm{~s}$ ) as $320 \times 240$ pixels per image, and analyzed with the program from the vendor. Average values were obtained for six observations (two recordings for three drop sites on the sample surface for each measurement). According to the supplier, the error of measurements is usually within $\pm 1^{\circ}$. However, it may be a bit higher in some cases. 


\section{Results and Discussion}

\subsection{NMR Study of the Extracted Substance in the Immersed Water}

To verify the hypothesis of the inner transport of the amphiphilic molecules inside the PDMS matrix, the substance in immersed water was extracted by lyophilizing the immersed water and further examined with ${ }^{1} \mathrm{H}$ NMR spectroscopy. The signals in the spectrum of the extracted powder from the Pluronic F127-embedded sample (marked by F127 + PDMS) is shown in Figure 2. As a reference, the NMR spectrum of pure Pluronic F127 was obtained as well (marked by Pluronic F127). At the same time, considering that there are lots of oligomers inside PDMS and that they might migrate as well, we also immersed the same amount of native PDMS in the water and lyophilized the immersed water. The extracted residue was sent for NMR study as a negative control (marked by Native PDMS). Comparing the three spectra, we discovered that the characterized peaks in Fluronic F127 could be found in that of the F127-embedded sample as well, which clearly suggest that the substance with molecules of Pluronic F127 migrated into the water. In the negative reference in our NMR study, a lot of peaks were found in the water from the native PDMS sample, which indicates that low-molecular-weight PDMS or its oligomer can migrate into water over time and further decrease its surface stability and performance. Comparing the spectra, these peaks from native PDMS could be found in the F127-embedded sample as well. Hence, the exact form of this lyophilized power is not clear. Possibly, it is the mixture of low-molecular-weight PDMS or its oligomer and Pluronic F127, or a composite of PDMS and Pluronic F127. However, it can be concluded that the Fluronic F127 molecules indeed move from PDMS after water treatment.

Figure 2. NMR study of lyophilized powder from the treated water.
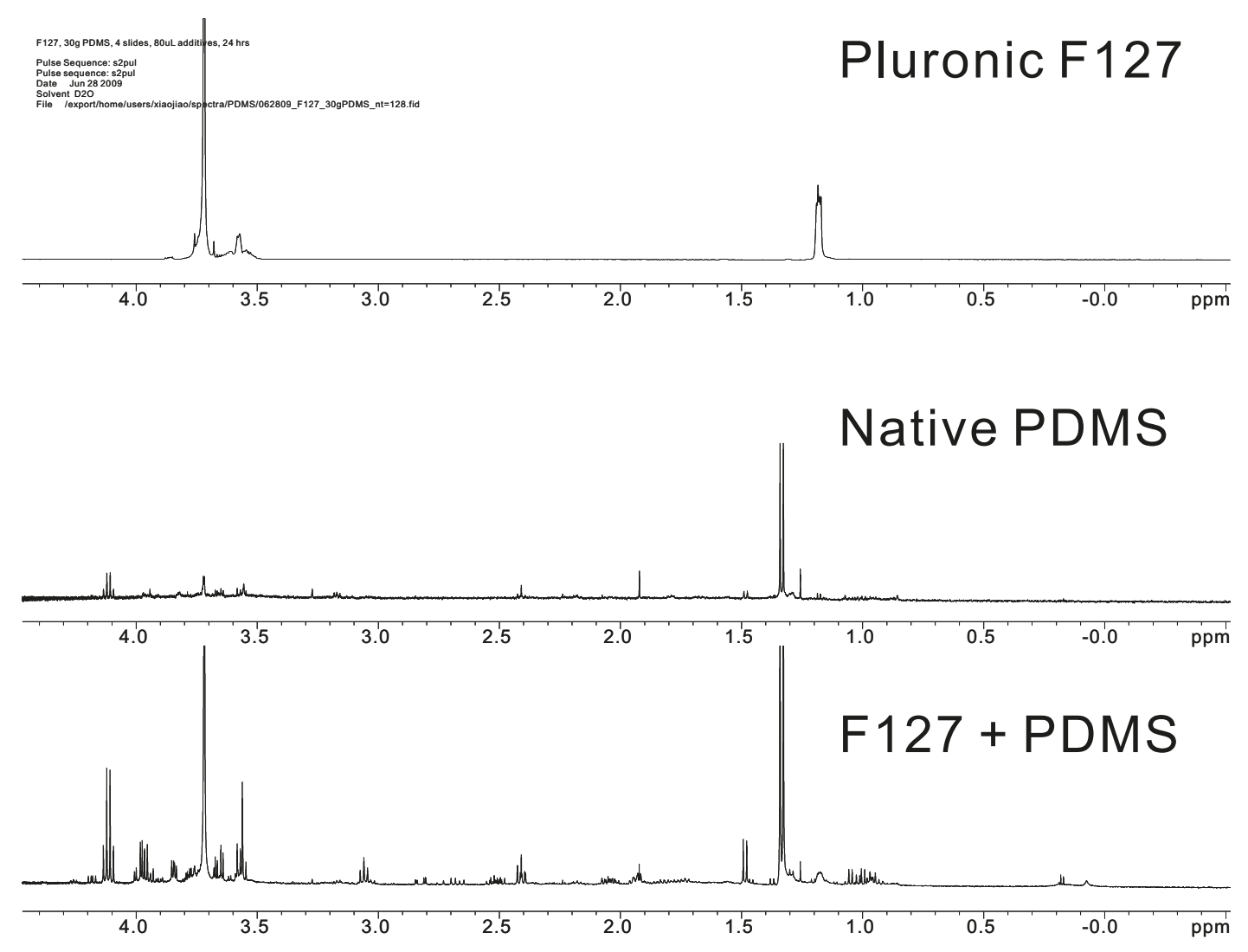


\subsection{Master Preparation and PDMS Replica}

Due to practical reasons in microfluidic device fabrication, the side closest to the master is the most useful surface of the device. That is, the surface property of the master is important during curing. In our case, we have the pluronic molecules in PDMS mixture. Reducing the total energy of the system, pluronic unimers migrate towards the interfaces, which include the liquid PDMS-ambient air and liquid PDMS solid interfaces, to help form the new surfaces. Hence, the properties of the interface would influence the molecule transport. Some of the pluronic will already be at the surface when the PDMS has been cured. Figure 3 shows continuous short-term measurements of the contact angles of the Pluronic F127-embedded surfaces replicated from different master materials. The one replicated from glass has a significantly lower contact angle curve than the rest at the same time.

As mentioned previously, when the surface interfaces with a hydrophilic environment, the pluronic has a tendency to migrate to the surface. One reasonable explanation is that the glass surface is hydrophilic with a lot of hydroxyl groups [23], thus favoring the similar groups (ethylene oxide) in Pluronic F127 to migrate and align towards the glass while the other three masters have more non-polar surfaces, which are not favoured to polar groups, e.g., ethylene oxide in pluronic. Hence, surfaces that have been cured against glass are more hydrophilic in the short term. Although the silicon wafer is usually covered with a very thin silica layer, it showed higher contact angles. The possible reason is that we used a very old wafer and applied a solvent-based cleaning product, which made the surface not as polar as that of glass. In a similar manner, the samples replicated from PDMS and polystyrene masters show higher initial contact angles than that from silicon, which could be due to the fact that hydrophobic PDMS and polystyrene help PDMS oligomers migrate towards the surface. Consequently, these migrated oligomers increase the hydrophobicity of the surface.

Figure 3. Short-term observations of the contact angles of the droplets on the surfaces replicated from different master materials.

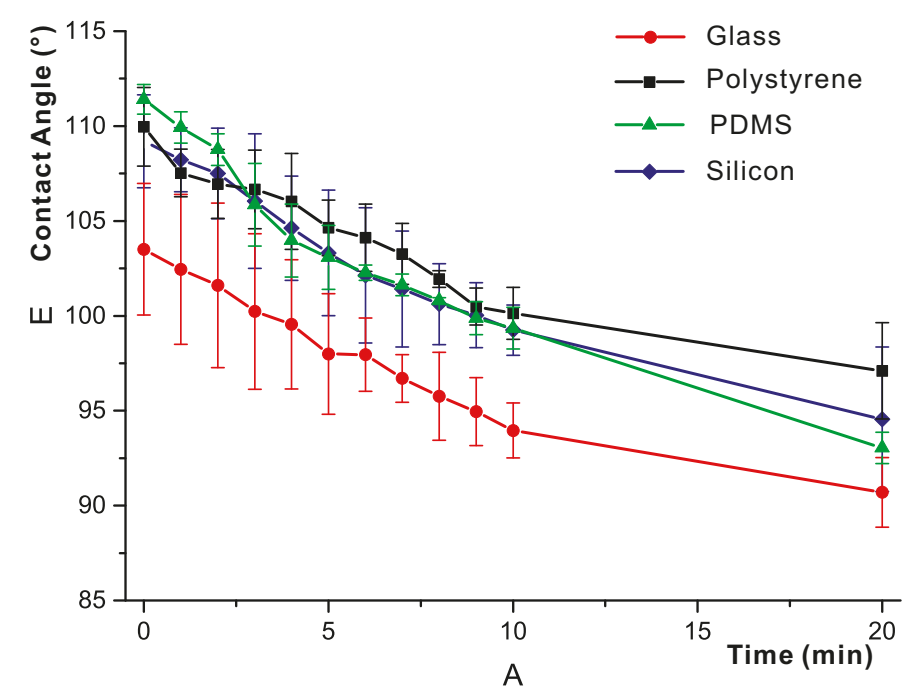

\subsection{Short-Term Study of the Effects of Water Exposure}

In order to understand the short-term behavior of the surface immersed in the water, continuous observations of the contact angles of the droplets on the surface were conducted. A droplet of 
de-ionized water was dispensed on the surface with Pluronic F127 (freshly peeled from the silicon master, without water exposure). The contact angles were measured continuously for $20 \mathrm{~min}$. To eliminate the influence of evaporation, a reference of native PDMS was made and measured as well (Figure 4). Significant lower contact angles were observed from the Pluronic F127-embedded sample at the same time point as compared to the native PDMS. This suggests that the surfactant interacts with the water and improves the surface hydrophilicity in the short term.

Figure 4. Short-term observations of the contact angles of the droplets on the surfaces.

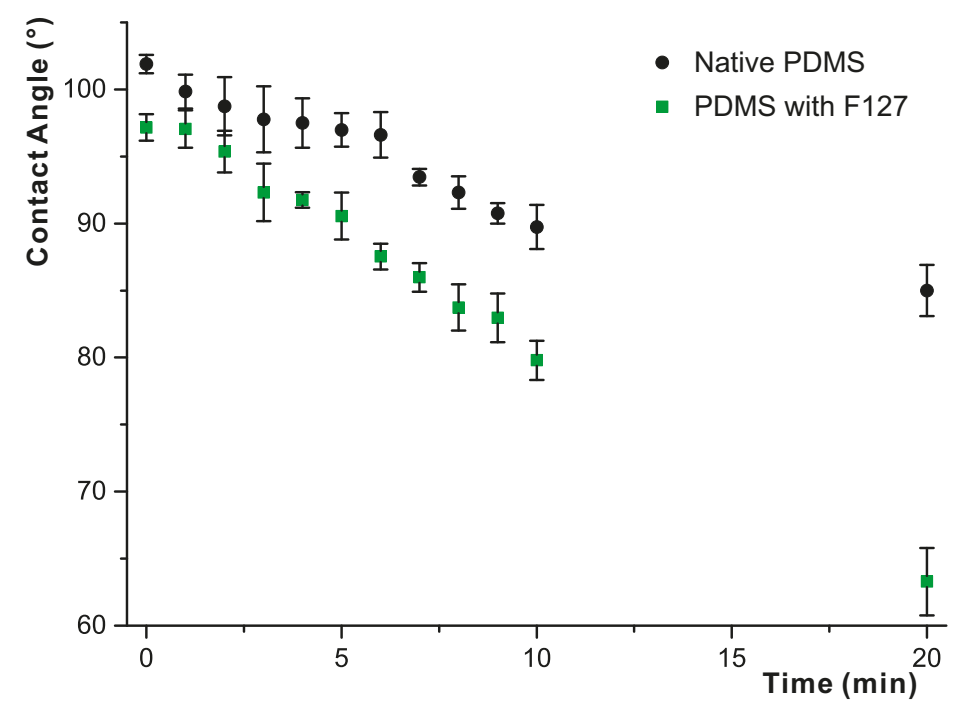

\subsection{Long-Term Study of the Effects of Water Exposure}

Since water exposure has a high impact on the performance of the surface, long-term immersion of the Pluronic F127-embedded PDMS in water was conducted (Figure 5). The behavior is not straightforward; the curve shows a trend that the contact angle of the modified sample first experiences a fast decrease and then slowly increases, which is quite different from that of native PDMS. Furthermore, from Figure 5, we found that long-term water exposure also has an impact on the wetting of native PDMS. It could be partly due to the flexibility of the PDMS backbone, where the oxygen groups were partly reorganized towards aqueous surface. The introduction of the amphiphilic molecules accelerates this processing. As pointed out in previous work, although pluronic cannot make a highly hydrophilic surface, it could significantly enhance the capability of bio-fouling resistance and decrease the potential damage of the cells [24].

Generally, temperature is an important parameter that can affect the mobility of all molecules. Four different temperatures $\left(25^{\circ} \mathrm{C}, 37^{\circ} \mathrm{C}, 54^{\circ} \mathrm{C}\right.$ and $\left.75^{\circ} \mathrm{C}\right)$ were selected in our study, as shown in Figure 6 . We discovered that the contact angle has the same trend at different temperatures as found above in the initial stage, whereby the contact angle ultimately increases, albeit slowly, after long-term water exposure (e.g., $500 \mathrm{~h})$.

To verify the versatility of the approach, other amphiphilic molecules were investigated, such as the similar Pluronic F68, PDMS-PO, and the commonly found surfactant Tween 80 (Figure 7). The chemical structures and molecular weights are summarized in Table 1. Similar to Pluronic F127, the contact angles of the other three samples became smaller and smaller at first and then increased after a 
critical point. The significant difference is that the occurrence of such a turning point varies greatly, which is related to their smaller molecular weights and shorter turning times. Unlike the pure PDMS or PDMS with Pluronic F127 or F68 at low concentration (when high concentration of F127 or F68 was added, it became slightly cloudy), when the other surfactants were co-cured with the prepolymer, the cured elastomers were not optically transparent, but rather cloudy and translucent (Figure 8). In particular, the sample with Tween 80 shows a wavy surface. It seems that the introduction of vinyl-functional Tween 80 to the uncured PDMS ultimately leads to their reaction with the prepolymer via covalent bonds [25] at the presence of Pt catalyst.

Figure 5. Measurements of the various contact angles with various water exposure times.

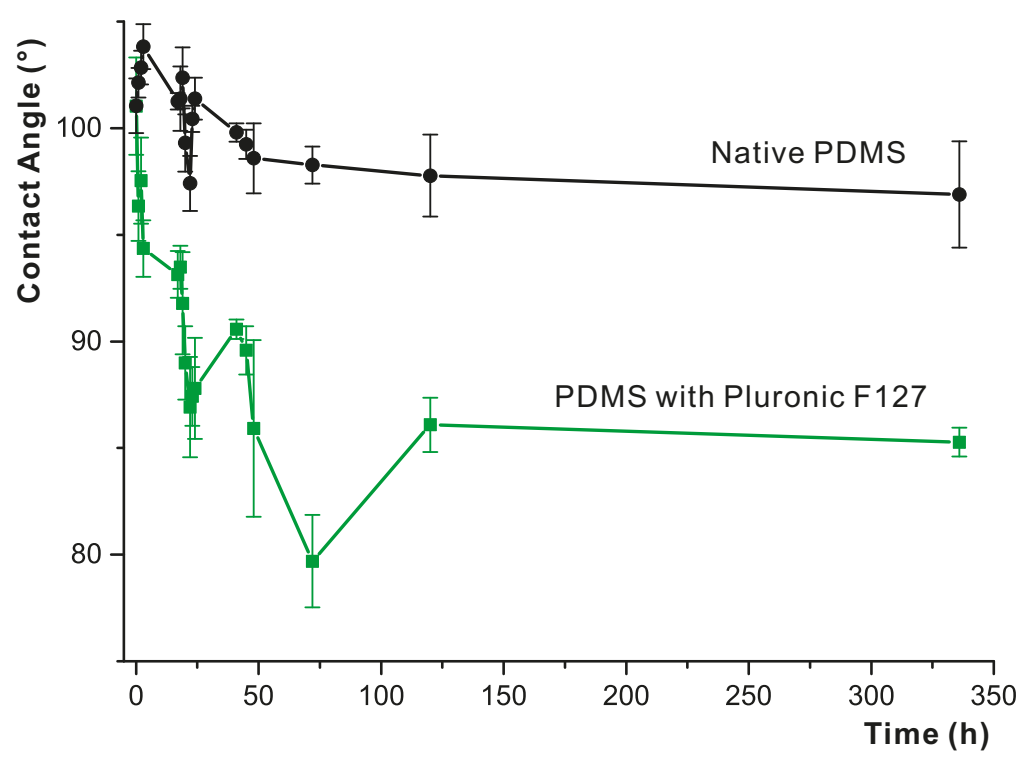

Figure 6. Measurements of the various contact angles with water exposure time under different temperatures with a F127-embedded PDMS slab from a silicon master.

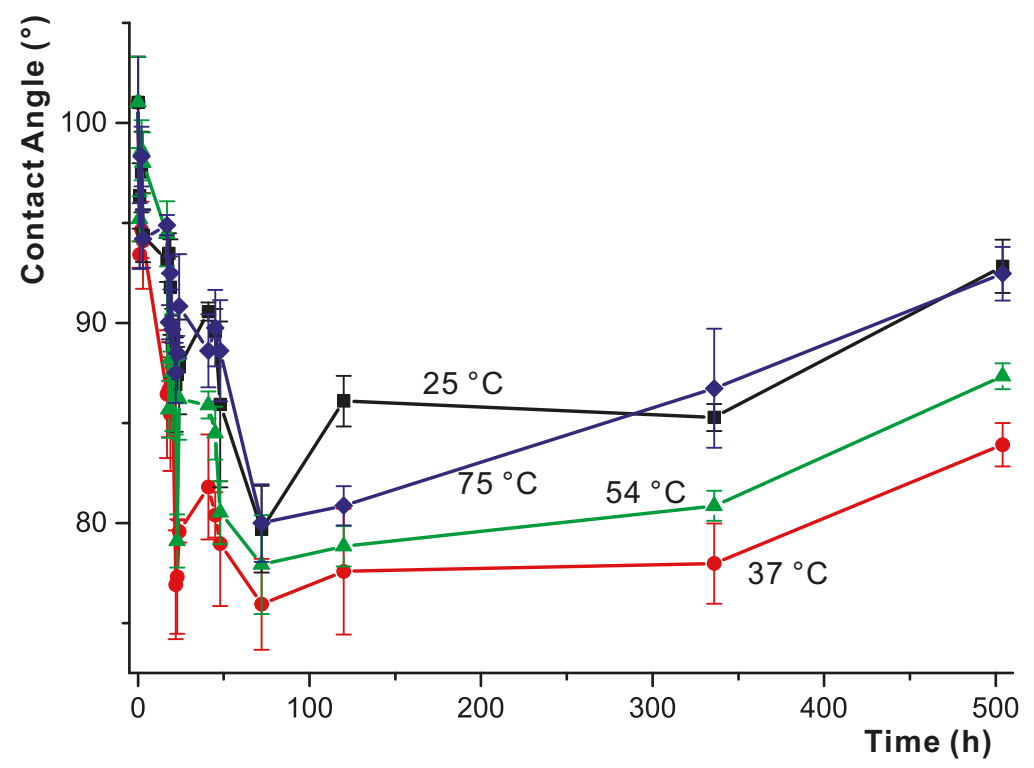


Table 1. The amphiphilic molecules used in the study.

\begin{tabular}{ccc}
\hline Amphiphilic Molecules & Chemical Structure & Molecular Weight $\left(\mathbf{M}_{\mathbf{w}}\right)$ \\
\hline Pluronic F127 & $(\mathrm{PEO})_{100}-(\mathrm{PPO})_{65}-(\mathrm{PEO})_{100}$ & $\sim 12,600$ \\
Pluronic F68 & $(\mathrm{PEO})_{78}-(\mathrm{PPO})_{30}-(\mathrm{PEO})_{78}$ & $\sim 8,400$ \\
PDMS-EO (AB111108) & $\mathrm{CH}_{3}\left[\mathrm{Si}\left(\mathrm{CH}_{3}\right)_{2} \mathrm{O}\right]_{n}-\left(\mathrm{C}_{2} \mathrm{H}_{5} \mathrm{O}\right)_{m} \mathrm{H}$ & $5,000-6,000$ \\
Tween80 & & 1,310 \\
\hline
\end{tabular}

Figure 7. Measured contact angles varying with water exposure time using different embedded amphiphilic molecules.

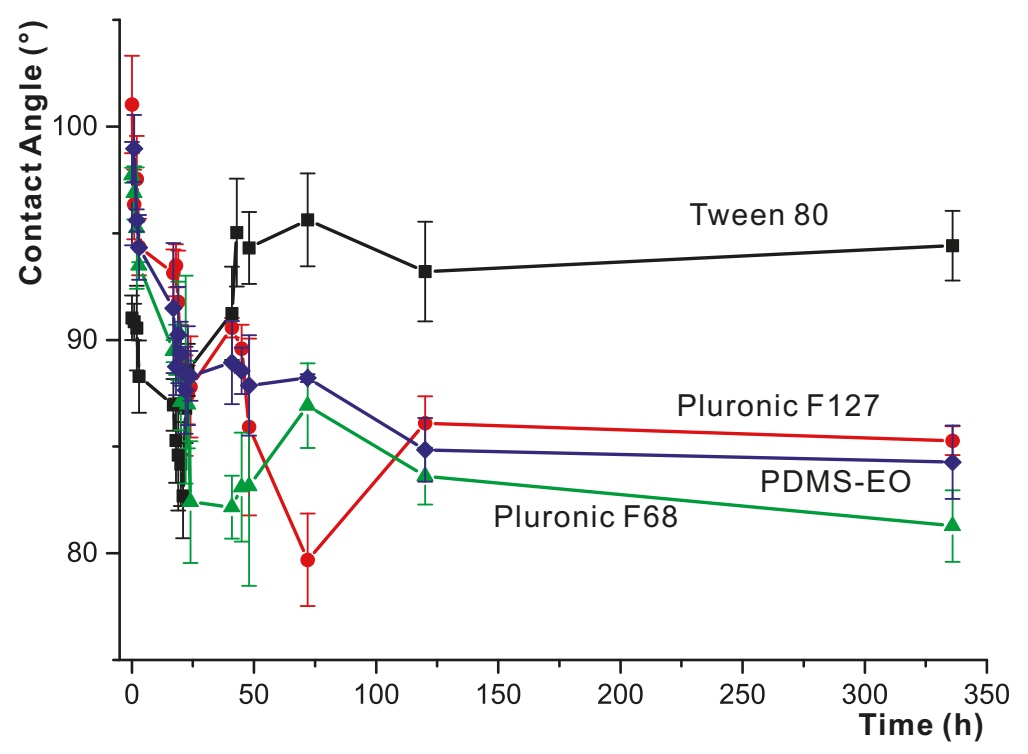

Figure 8. Photos of the cured PDMS with different molecules: (a) native PDMS; (b) with Pluronic F127; (c) PDMS with Tween 80; and (d) PDMS with PDMS-EO.

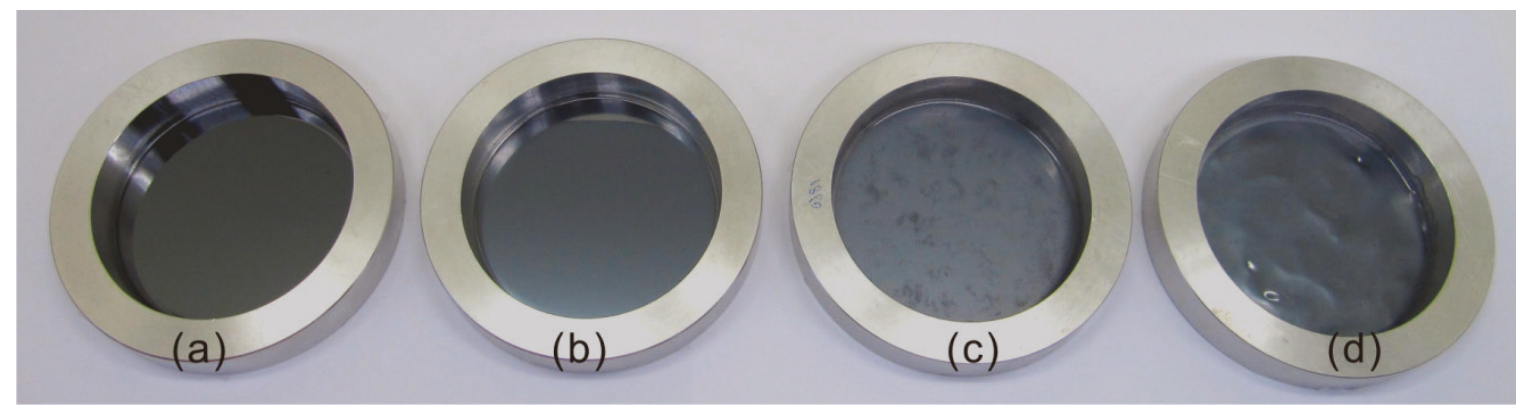

\subsection{Interpretation of the Interactions between the Embedded Amphiphilic Molecules and PDMS Matrix}

As mentioned in the Introduction, Pluronic F127 is a triblock copolymer comprising a hydrophobic part, poly(propylene oxide) (PPO) in the middle and a hydrophilic part, poly(ethylene oxide) (PEO) at both ends of the PPO. The existence of hydrophobic parts increases the probability of hydrophobic interaction with PDMS, while hydrophilic parts bring the opportunity of increasing hydrophilic wetting by introducing a hydrophilic portions domain outward interface. The Pluronic F127 solution 
was prepared in ethanol, where the block copolymer easily formed complex micelles with very low critical micellization concentration (CMC) [26], together with unimers in the solution.

When a small amount (e.g., $80 \mu \mathrm{L}$ ) of Pluronic F127 solution was added to the (e.g., $12.00 \mathrm{~g}$ ) prepolymer of PDMS, the unimers and micelles of Pluronic F127 were surrounded by the very hydrophobic environment of the host prepolymer-PDMS. The original micelles from the ethanol-based solution, with PPO parts inside and PEO parts as outward ethanol molecules, were dissolved in the hydrophobic environment. A rearrangement of Pluronic F127 unimers or a formation of a new type of micelle would follow. However, since such a rearrangement happened together with the curing process of PDMS, the process might be frozen in a transitional state. Furthermore, as an amphiphilic additive in PDMS, Pluronic F127 has a tendency to migrate to the surface. This means that there may be some Pluronic F127 molecules on the cured PDMS surface (surface-embedded), which are not in the ideal configuration for yielding high hydrophilicity, since the molecule is configured during the curing process, where there is low or no hydrophilic interaction. These configurations rearrange to the optimal configuration in the presence of water; which is when the hydrophobic part, the PPO, is at the surface but embedded in the PDMS while the hydrophilic part, PEO, is stretched out from the surface. We believe that this is the main reason why we observed a decreasing curve in the short-term study of the contact angle in Figure 4.

If we make the time span longer, Pluronic F127 molecules will migrate to the aqueous environment due to a very high gradient of solubility. At the same time, some of the molecules on the surface leach into the water. To better understand the interaction between the embedded molecules and the surface, and make the system simpler, we exclude the reorientation of the PDMS backbone since it cannot generate the ideal surface to inhibit bio-fouling or enhance cell viability. The two competitive processes molecules adsorbed to the surface from the bulk and desorbed from the surface to the water to dominate the behavior of the surface. According to Figures 5-7, the contact angle first experiences a fast decrease, then a turning point appears, and after that a slow increase follows, as summarized in Figure 9. Our interpretation is that this results from the competition between three interaction mechanisms: Pluronic F127, PDMS, and water. It could be categorized into three stages according to the dominant mechanism. Initially, in the first stage (Stage I), upon immersing in water, embedded molecule transport towards the surface was triggered. Although the speed is high, due to the steep gradient, the number of the arriving molecules from the bulk is small. Hence, the reconfiguration and alignment of the surface-embedded Pluronic F127 plays a most important role on the wetting. Consequently, it shows a rapid contact angle decrease in a short time span. In the second stage (Stage II), the arriving molecules from the bulk are dominant, increasing the number of adsorbed molecules at the surface. During this stage, the gradient between the PDMS matrix and surface is still steep, which drives the transport of embedded molecules strongly towards the surface. When the other gradient (between surface and water) is not as steep, the molecule transported into water is not as influential. Consequently, the contact angle decreases continuously. However, the continuously arriving embedded molecules decrease the gradient between the PDMS matrix and the surface and increase the gradient between the surface and the water. Hence a turning point appears at the end of Stage II. If we could optimize the process and freeze the transport to the dry condition at the end of this stage, it might produce a very high performance surface. In principle, this surface could be kept a while and regenerated when immersed in water again after longer term storage in dry conditions. However, more 
investigations are needed for further verification. Finally, in Stage III, the trend is inversed thoroughly. The surface shows higher and higher contact angles and finally reached a relative stable contact angle over time, since without removing the immersed water, a dynamic coating will form in the aqueous environment. There, the pluronic molecules will always come back to the PDMS and leave it in a continuous way, reaching a relatively stable state with two opposite forces that nearly balance each other. In short, the variation with time of surface coverage (i.e., surface concentration) of embedded amphiphilic molecules and their rearrangement on the surface will determine the surface performance and contact angle.

In addition, there may be for a period an intermediate stage between Stage (II) and (III), where there is a balance between arriving molecules from the bulk and adsorbed molecules. Finally, the contact angle measurement alone cannot provide direct information regarding to the mechanism of interaction of water with the molecule embedded in PDMS. Hence, it will be better to introduce some other more advanced technologies for future further investigations, such as a spectroscopic technique that could help with the interpretation of the molecular interactions on the bulk and surface of the PDMS.

Figure 9. Possible mechanism of molecule transport in the PDMS matrix and surface behavior.

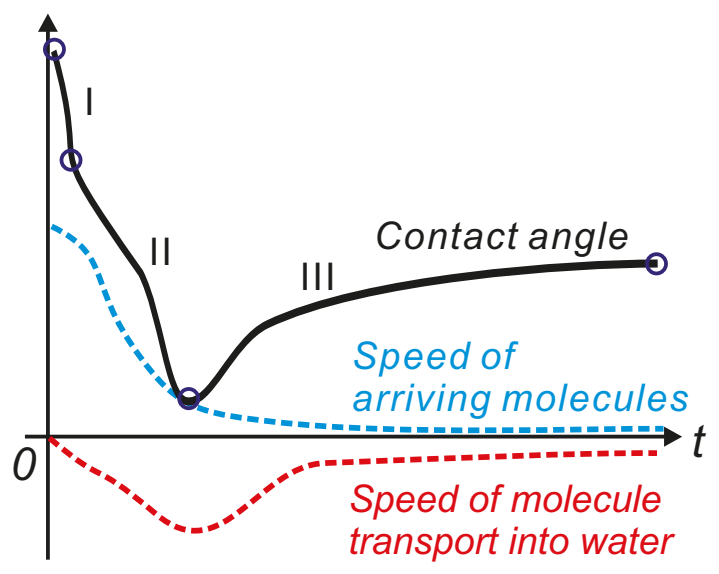

\section{Summary}

In this work, by varying the processing conditions, the transport of embedded amphiphilic molecules in the PDMS was systematically studied by measuring the wetting angle along with time. The results indicate that the curing master materials, water exposure time, temperature, and type and size of molecules also impact the transport process. In particular, the water exposure time clearly plays an important role in the surface properties. Our interpretation is that molecular rearrangement of the surface-embedded molecules improves surface coverage in the short term, while at a longer term transport of molecules embedded in the bulk enhance its coverage, which should be quantitatively proved in future work.

\section{Acknowledgments}

The authors thank Karin D. Caldwell and Jonas Bergquist, Department of Chemistry BMC, Uppsala University for valuable suggestions. Zhigang Wu holds a Junior Researcher position at the Swedish Research Council (Contract No. 621-2010-5443). 


\section{Author Contributions}

Wenjun Qiu performed most of the experiments, Xiaojiao Sun conducted NMR characterization, Wenjun Qiu, Chaoqun Wu, Klas Hjort, Zhigang Wu analyzed the data, Wenjun Qiu, Klas Hjort, Zhigang $\mathrm{Wu}$ wrote the manuscript, Zhigang $\mathrm{Wu}$ supervised the work.

\section{Conflicts of Interest}

The authors declare no conflict of interest.

\section{References}

1. Duffy, D.C.; McDonald, J.C.; Schueller, O.J.A.; Whitesides, G.M. Rapid prototyping of microfluidic systems in poly(dimethylsiloxane). Anal. Chem. 1998, 70, 4974-4984.

2. Makamba, H.; Kim, J.H.; Lim, K.; Park, N.; Hahn, J.H. Surface modification of poly(dimethylsiloxane) microchannels. Electrophoresis 2003, 24, 3607-3619.

3. Wong, I.; Ho, C.H. Surface molecular property modifications for poly(dimethylsiloxane) (PDMS) based microfluidic devices. Microfluid. Nanofluidics 2009, 7, 291-306.

4. Zhou, J.W.; Ellis, A.V.; Voelcker, N.H. Surface modification for PDMS-based microfluidic devices. Electrophoresis 2010, 33, 89-104.

5. Mukhopadhyay, R. When PDMS isn't the best. Anal. Chem. 2007, 79, 3248-3253.

6. Hillborg, H.; Ankner, J.F.; Gedde, U.W.; Smith, G.D.; Yasuda, H.K.; Wikstrom, K. Crosslinked polydimethylsiloxane exposed to oxygen plasma studied by neutron reflectometry and other surface specific techniques. Polymer 2000, 41, 6851-6863.

7. Wu, Z.G.; Hjort, K.; Wicher, G.; Svenningsen, A.F. Microfluidic high viability neural cell separation using viscoelastically tuned hydrodynamic spreading. Biomed. Microdevices 2008, 10, 631-638.

8. Hedlund, E.; Pruszak, J.; Ferree, A.; Vinuela, A.; Hong, S.; Isacson, O.; Kim, K.S. Selection of embryonic stem cell-derived enhanced green fluorescent protein-positive dopamine neurons using the tyrosine hydroxylase promoter is confounded by reporter gene expression in immature cell populations. Stem Cells 2007, 25, 1126-1135.

9. Lee, J.N.; Park, C.; Whitesides, G.M. Solvent compatibility of poly(dimethylsiloxane) based microfluidic devices. Anal. Chem. 2003, 75, 6544-6554.

10. Alexandridis, P.; Hatton, T.A. Poly(ethylene oxide) poly(propylene oxide) poly(ethylene oxide) block copolymer surfactants in aqueous solutions and at interfaces: Thermodynamics, structure, dynamics, and modelling. Colloids Surface 1995, 96, 1-46.

11. Singh-Joy, S.D.; McLain, V.C. Safety assessment of poloxamers 101, 105, 108, 122, 123, 124 , $181,182,183,184,185,188,212,215,217,231,234,235,237,238,282,284,288,331,333$, $334,335,338,401,402,403$, and 407, poloxamer 105 benzoate, and poloxamer 182 dibenzoate as used in cosmetics. Int. J. Toxicol. 2008, 27, 93-128.

12. Li, J.T.; Carlsson, J.; Huang, S.C.; Caldwell, K.D. Adsorption of Poly(ethylene oxide)-Containing Block Copolymers a Route to Protein Resistance. In Hydrophilic Polymers; Glass, J.E., Ed.; American Chemical Society: Washington, DC, USA, 1996; pp. 61-78. 
13. Jeon, S.I.; Lee, J.H.; Andrade, J.D.; de Gennes, P.G. Protein-Surface interactions in the presence of polyethylene oxide: I. Simplified theory. J. Colloid Interface Sci. 1991, 142, 149-158.

14. Jeon, S.I.; Andrade, J.D. Protein-Surface interactions in the presence of polyethylene oxide: II. Effect of protein size. J. Colloid Interface Sci. 1991, 142, 159-166.

15. Ottenbrite, R. Frontiers in Biomedical Polymer Applications; Technomic Publishing Co.: Lancaster, UK, 1999.

16. Kabanova, A.V.; Alakhovb, V.Y. Pluronic ${ }^{\circledR}$ block copolymers as novel polymer therapeutics for drug and gene delivery. J. Control. Release 2002, 82, 189-212.

17. Batrakova, E.V.; Alakhovb, V.Y. Pluronic block copolymers evolution of drug delivery concept from inert nanocarriers to biological response modifiers. J. Control. Release 2009, 130, 98-106.

18. Nagant, C.; Savage, P.B.; Dehaye, J.P. Effect of pluronic acid F-127 on the toxicity towards eukaryotic cells of CSA-13, a cationic steroid analogue of antimicrobial peptides. J. Appl. Microbiol. 2012, 112, 1173-1183.

19. Khattak, S.F.; Bhatia, S.R.; Roberts, S.C. Pluronic F127 as a cell encapsulation material: utilization of membrane-stabilizing agents. Tissue Eng. 2005, 11, 974-983.

20. Luk, V.N.; Mo, G.C.H.; Wheeler, A.R. Pluronic additives: A solution to sticky problems in digital microfluidics. Langmuir 2008, 24, 6382-6389.

21. Wu, Z.G.; Hjort, K. Surface modification of PDMS by gradient-induced migration of embedded Pluronic. Lab Chip 2009, 9, 1500-1503.

22. Zeng, X.; Xu, G.; Gao, Y.; An, Y. Surface wettability of (3-aminopropyl)triethoxysilane self-assembled monolayers. J. Phys. Chem. B 2010, 115, 450-454.

23. Hjerten, S. High-performance electrophoresis: Elimination of electroendosmosis and solute adsorption. J. Chromatogr. A 1985, 347, 191-198.

24. Li, J.T.; Caldwell, K.D.; Rapoport, N. Surface properties of pluronic-coated polymeric colloids. Langmuir 1994, 10, 4475-4482.

25. Thompson, D.B.; Fawcett, A.S.; Brook, M.A. Simple Strategies to Manipulate Hydrophilic Domains in Silicones. In Silicon Based Polymers Advances in Synthesis and Supramolecular Organization; Ganachaud, F., Boileau, S., Bourt, B., Eds.; Springer: Amsterdam, The Nethrelands, 2008; pp. 29-38.

26. Myers, D. Surfactant Science and Technology, 3rd ed.; Wiley-Interscience: Hoboken, NJ, USA, 2005.

(C) 2014 by the authors; licensee MDPI, Basel, Switzerland. This article is an open access article distributed under the terms and conditions of the Creative Commons Attribution license (http://creativecommons.org/licenses/by/3.0/). 